\title{
Ruy Mauro Marini y el subimperialismo brasileño en Bolivia y Paraguay en el siglo XXI
}

Fernando Carnebia López ${ }^{1}$

Rafael Teixeira de Lima

Resumen: En el presente artículo son retomados los trazos centrales en la construcción de la categoría subimperialismo que realiza Ruy Mauro Marini en la década de 1960-70, a partir de los cuales son presentados elementos que permiten visualizar la actualidad de dicha conceptualización para comprender la proyección de la economía y del estado brasileño sobre Bolivia y Paraguay. En el caso de Bolivia son expuestos algunos elementos acerca de los proyectos de integración de infraestructura y su relación con la expansión de la soja, así como la relación de ambos con los intereses brasileños presentes en dicho país. Para el caso paraguayo, son desarrollados de forma breve los principales ejes sobre los que se articula la proyección del subimperialismo brasileño, siendo estos: la soja y la presencia de los denominados brasiguayos, la energía eléctrica e Itaipu y la diferenciación de la pauta de comercio bilateral, que permitiría vislumbrar una división regional del trabajo.

Palabras clave: Marini; subimperialismo; dependencia.

Abstract: In this article are retaken the central lines in the construction of the subimperialism category made by Ruy Mauro Marini in the 60's and $70^{\prime}$ s, from which are presented elements that shows the relevance

\footnotetext{
${ }^{1}$ Estudiante de Maestría del Programa de Posgrado en Integración Contemporánea de América Latina (PPGICAL) de la Universidad Federal de la Integración Latinoamericana (UNILA). E-mail: fernando.carnebia@gmail.com.

${ }^{2}$ Estudiante de Maestría del Programa de Posgrado en Integración Contemporánea de América Latina (PPGICAL) de la Universidad Federal de la Integración Latinoamericana (UNILA). E-mail: rafatdelima@gmail.com.
} 
of such concepts to understand Brazilian's economy and Brazilian's state projection on Bolivia and Paraguay. In the case of Bolivia are exposed some elements about infraestructure integration projects and their relation to the expansion of soybean as well as their relationship with Brazilian interests in that country. For the case of Paraguay, are developed briefly the main axes on which Brazilian's subimperialism projection is articulated, these being: soy and the presence of so-called brasiguayos, electrical energy and Itaipu and the differentiation of the bilateral trade pattern, that would envision a regional division of labor. Keywords: Marini; subimperialism; dependency.

\section{El subimperialismo brasileño para Ruy Mauro Marini}

Considerando la llegada del capitalismo a la fase de los monopoliosy el capital financiero (LENIN, 2011) y que unos de sus trazos distintivos es la exportación de capitales, se destaca que a comienzos del siglo XX, la forma predominante de ello son los préstamos. Por otra parte, en el post-segunda guerra mundial con la llegada de Estados Unidos a la posición de potencia hegemónica dentro del capitalismo, la forma de exportación de capital predominante pasa a ser la inversión extranjera directa, lo que genera un mayor entrelazamiento de la producción a nivel mundial.

Es a partir de ello que Ruy Mauro Marini señala que durante el período de la hegemonía británica se crearía y se consolidaría el mercado mundial, durante el período de la hegemonía estadounidense sería el de la "[...] integración imperialista de los sistemas de producción" (MARINI, 1977, p. 3). El proceso de integración imperialista de los sistemas de producción genera una reconfiguración de la División Internacional del Trabajo (DIT) en la cual "son transferidas para los países dependientes etapas inferiores de la producción industrial" (MARINI, 1973). Asimismo, según Luce, este proceso genera las bases materiales sobre las cuales se desarrollará el subimperialismo. Luce afirma que el subimperialismo es una

\begin{tabular}{l|l}
\hline 70 & Ruy Mauro Marini y el Subimperialismo...
\end{tabular} 
[...] explicación desarrollada por Ruy Mauro Marini para el fenómeno emergido en los años 1960-70, en el contexto de una nueva división internacional del trabajo que se desarrolla en el post-segunda guerra mundial y de la conformación de subcentros económicos y políticos de la acumulación mundial que de ella devienen (LUCE, 2011, p. 16).

Para Marini, del mismo modo que el subdesarrollo no era considerado una etapa previa al desarrollo, sino una forma específica de capitalismo, un capitalismo sui generis, el cual está dentro de la dinámica de producción y reproducción del sistema; el imperialismo no es visto de forma disociada de la dependencia, sino por el contrario:

Para a teoria da dependência [...] o imperialismo não é algo externo à dependência. Pelo contrário, o imperialismo permeia toda a economia e a sociedade dependentes, representando um fator constitutivo de suas estruturas sócio-econômicas, de seu Estado, de sua cultura (MARINI, 1992, p. 90).

Sin embargo, el desarrollo del capitalismo en su forma monopolista en los países centrales, permitió que la expansión de capitales a regiones periféricas promuevan una industria incipiente en algunos de esos países, favorecida por la II Guerra Mundial y el redireccionamiento de esfuerzos de las grandes potencias relacionadas al comercio internacional. Esa nueva dinámica favorecería la configuración del subimperialismo.

Como quiera que sea, la expansión y aceleración tanto de la circulación de capital productivo como de la circulación del capital dinero han ido configurando una nueva economía mundial capitalista, que reposa sobre un esquema de división internacional de trabajo distinto al que regía antes de la crisis 
mundial que mencionamos inicialmente. Pasó el tiempo del modelo simple centro-periferia, caracterizado por el intercambio de manufacturas por alimentos y materias primas. Nos encontramos ante una realidad económica en que la industria asume un papel cada vez más decisivo. Esto es cierto aún cuando el capital industrial se amplía y fortalece en áreas extractivas y agrícolas; lo que es todavía más, cuando consideramos la extensión y diversificación a escala mundial de la industria manufacturera. El resultado ha sido un reescalonamiento, una jerarquización de los países capitalistas en forma piramidal y, por consiguiente, el surgimiento de centros medianos de acumulación - que son también potencias capitalistas medianas -, los que nos ha llevado a hablar de la emergencia de un subimperialismo. (MARINI, 1977, p. 8).

Los sectores vinculados al capital extranjero se encuentran en una situación privilegiada ya que cuentan con condiciones de producción más favorables debido al mayor nivel tecnológico de los medios de producción (especialmente maquinaría) obtenida en el extranjero lo que permite aumentar la productividad y con ello apropiarse de plusvalía extraordinaria.

En esa línea, al entender el subimperialismo como la forma que asume la economía dependiente al llegar a la fase de los monopolios y del capital financiero, lo cual es fruto de una profundización de la tendencia a la concentración y centralización de capital, Marini indica dos componentes básicos de su constitución, los cuales son: una composición orgánica media en la escala mundial de los aparatos productivos nacionales y el ejercicio de una política externa expansionista relativamente autónoma (MARINI, 1977, p. 17).

De ello se desprende la categoría cooperación antagónica, la cual, en términos de política externa, propicia una expansión de la economía del país dependiente con respecto a otras economías

\begin{tabular}{l|l}
\hline 72 & Ruy Mauro Marini y el Subimperialismo...
\end{tabular} 
dependientes, siempre y cuando se desarrolle dentro del marco de la hegemonía imperialista. Para Luce (2011), una política expansionista subimperialista tiene dos trazos esenciales: la búsqueda de una hegemonía regional y de una autonomía relativa frente al imperialismo dominante. Estos dos elementos son fundamentales para asegurar condiciones favorables a la reproducción del capital en la economía subimperialista.

Zibechi, por otra parte, al analizar los aspectos de la construcción del subimperialismo brasileño, dice que su política expansionista teorizada por la Escuela Superior de Guerra (ESG) tenía entre sus propuestas la alianza con los Estados Unidos contra el comunismo, la expansión interna hasta la Amazonia, para ocupar "espacios vacíos" del territorio brasileño y la expansión externa hasta el Pacífico para cumplir con el "destino manifiesto" del Brasil, teniendo como objetivo general que nortea todos esos intentos, el control del Atlántico Sur (ZIBECHI, 2012, p. 36). De esa forma, la idea de Brasil potencia, estaba en el horizonte de los geopolíticos y formuladores de políticas de las décadas 1960 y 1970.

El fortalecimiento del prestigio nacional brasileño en el ámbito externo era visualizado, en aquel momento, con el objetivo de proyectar internacionalmente las singularidades de ese país en el contexto de los países emergentes, particularmente de los suramericanos. De esta forma, Brasil aceptaba la hegemonía norteamericana en el Atlántico Sur, pero teniendo reservado su derecho "inalienable" de ejercerla en el futuro (RAMANZINI JR \& VIGEVANI, 2012, p. 125).

Marini describe la posición brasileña en aquel momento como direccionada a "[...] convertirse en el centro de irradiación de la expansión imperialista en América Latina, creando inclusive las premisas de un poderío militar propio" (MARINI, 1974, p.74). La idea, formulada por la ESG, de que Brasil debería "o expandirse o perecer" ganó notoriedad en el conjunto de la sociedad brasileña, alcanzando sectores de la burguesía nacional que buscaban el estrechamiento de relaciones con el Estado (ZIBECHI, 2012, p. 41). 
La existencia de una cooperación antagónica dada por la relación entre la superpotencia hegemónica y un país medianamente desarrollado posibilita la creación de "[...] una simbiosis entre los intereses de la gran industria y los sueños hegemónicos de la elite militar (MARINI, 1974, p. 71). Las burguesías nacionales, conscientes de su incapacidad de competir en mismo grado de importancia y influencia de los centros imperialistas, buscaban, mediante asociaciones con el Estado nacional, organizar sus fuerzas y obtener ventajas con el proyecto subimperialista (MARINI, 1977, p. 21).

Tomando como base el momento de reconfiguración de la división internacional del trabajo y del surgimiento de nuevos centros que pasan a ocupar una posición intermedia entre el centro y la periferia (PRECIADO, 2008; LUCE, 2011, p. 1) se discute la existencia de un proyecto de la dictadura militar brasileña que vinculaba su política externa a los intereses de los Estados Unidos, con la intención de asumir posición de potencia regional en América del Sur (ZIBECHI, 2012, p. 31).

\section{Subimperialismo como un patrón de reproducción del capital}

El subimperialismo, al recrear la dependencia, crea un patrón de reproducción del capital que le es propio. En palabras de Luce,

[...] el subimperialismo, al reactivar la dinámica del capital en la combinación de factores descriptos por sus nexos categoriales, se articula como un patrón específico de reproducción del capital, dándole forma subimperialista. En otras palabras, las contra-tendencias puestas en marcha para restaurar la unidad entre producción y realización son la síntesis de la lógica subimperialista, cuyos trazos característicos cumplen una función en el sentido de asegurar la reproducción del capital en escala ampliada (LUCE, 2011, p. 34). 
Ese patrón de reproducción del capital subimperialista se manifiesta a través de un esquema de realización propio, el cual está sustentado en tres elementos: el establecimiento de una sociedad de consumo (sui generis), el aumento de la capacidad de compra del Estado y la proyección en el mercado externo. El establecimiento de una sociedad de consumo, principalmente consumo suntuoso, es posible debido a una distribución de la renta fuertemente regresiva que permite el consumo por parte de las camadas medias y altas, en detrimento de las camadas más pobres. Este consumo suntuoso garantiza mercado para la industria de alto nivel tecnológico y profundiza la disociación entre la producción y el consumo de las grandes masas.

El aumento de la capacidad de compra del Estado se da fundamentalmente a través de la realización de infraestructura, con lo cual se genera una expansión del mercado de bienes de capital. Por su parte, la proyección en el mercado externo se da, inicialmente, a través de la exportación de manufacturas (posteriormente capitales).

En síntesis, la combinación del mercado externo, el Estado y el consumo suntuoso- combinado con una distribución regresiva de la renta son los elementos que componen el denominado esquema de realización del subimperialismo, el cual se configura como la válvula de escape que permite restaurar la unidad entre la producción y la realización del valor que a comienzo de la década de 1960 se encontraba en crisis en Brasil.

Mathias Luce sintetiza la idea de Marini del esquema de realización del subimperialismo en las décadas de 1960 y 1970 a partir de cuatro variables: la composición orgánica media nacional del capital, con la presencia con cierto grado de operación de capital financiero y de los monopolios capitalistas en la economía dependiente; el esquema tripartito de realización de mercancías (mercado externo, Estado y consumo suntuario); la hegemonía en un subsistema regional de poder para garantizar el desarrollo del sector de bienes durables 
en una economía dependiente y, por fin, la política de cooperación antagónica de un país dado con relación al imperialismo dominante (LUCE, 2011, p. 14).

De forma complementaria (aunque existan diferencias) a las variables sintetizadas por Luce, que tiene un enfoque más económico, Zibechi afirma, poniendo un mayor énfasis en la proyección de poder que era parte del proyecto de política externa de Brasil, que la tesis del subimperialismo se articulaba en tres ejes,:

[...] la hegemonía absoluta de los Estados Unidos en el mundo, la existencia de centros medianos de acumulación, dependientes del centro, que matenían una relación de cooperación antagônica con Estados Unidos y que a la vez praticaban formas de expansionismo en la región, y la existencia de un proyecto político subimperialista que de alguna manera encarnaba la ditadura militar (ZIBECHI, 2012, p. 42).

A modo de síntesis, puede verse que

[...] el subimperialismo, en Marini, es producto histórico de las contradicciones provocadas en el capitalismo brasileño, como consecuencia de la nueva fase de la división internacional del trabajo, marcada por la diferenciación interna de la burguesía y por la lógica de la cooperación antagónica y el impasse político que resultó de la amalgama de todos esos factores, en el curso de la crisis de realización los años 60, que reflejaba las leyes propias de la economía dependiente, en particular las contradicciones verificadas en el ciclo del capital (LUCE, 2011, p. 22).

Con el fin de visualizar la actualidad de la tesis del subimperialismo desarrollada por Marini, en las próximas sesiones son presentados algunos elementos que contribuyen a comprender la

\begin{tabular}{l|l}
\hline 76 & Ruy Mauro Marini y el Subimperialismo...
\end{tabular} 
proyección del subimperialismo brasileño sobre los vecinos Bolivia y Paraguay.

\section{Impactos en Bolivia}

Teniendo en cuenta la conceptualización del subimperialismo para Ruy Mauro Marini, se busca presentar algunos ejemplos de la actuación de Brasil que permiten discutir la validez de esta categoría, así como la contraposición entre la posibilidad de una expansión autónoma frente a los dictados del imperialismo hacia la construcción de un liderazgo solidario para la región y, por otro lado, la reafirmación de un subimperialismo revitalizado y adaptado a pleno siglo XXI.

Si Marini teoriza el subimperialismo en el exilio, en el marco de las grandes hostilidades proporcionadas por la dictadura militar brasileña, Zibechi (2012) trae un ejemplo de cómo la intervención brasileña estaba dando lugar a la consolidación del golpe de Estado del general Hugo Bánzer en Bolivia, contra el gobierno de Juan José Torres, en agosto de 1971:

La intervención en Bolivia se asentó en dos tesis en ese momento en boga entre los militares brasileños: "la doctrina del cerco", que decía que Brasil estaba rodeado de regímenes hostiles, y la "guerra ideológica preventiva" para neutralizar esa situación. El golpe partió de Santa Cruz, donde ya se asentaban poderosos empresarios brasileños, convertida en la base territorial de los golpistas [...] El 15 de agosto, cuatro días antes del golpe, Brasil había declarado la movilización de tropas en la frontera; los aviones que llevaban armas a militares y civiles golpistas ostentaban la bandera brasileña (ZIBECHI, 2012, p. 37).

Tales esfuerzos nacionales emprendidos en Bolivia traerían uma recompensa: los acuerdos comerciales que aseguraban a Brasil precios 
preferenciales en la compra de petróleo, gas, manganeso y mineral de hierro (QUIROGA, 1982). Con tales acuerdos y la garantía de fuentes seguras de materias primas, el proyecto Brasil Potencia podría desarrollar otras medidas, como líneas de comunicación con el Pacífico, con la construcción de un ferrocarril entre Santa Cruz y Cochabamba, dentro de un proyecto más amplio de interconexión entre Santos, en el Atlántico, y Arica, en el Pacífico.

Zibechi (2012) señala con gran precisión que tales objetivos no saldrían de las exportaciones brasileñas a la región, pero serían retomados décadas después bajo otro nombre: Iniciativa para la Integración de la Infraestructura Regional Suramericana (IIRSA) .

La reciente expansión de megaproyectos de infraestructura y de interconexión física por el territorio boliviano ha estado acompañada por el rápido crecimiento de la extracción vegetal y mineral, que avanza sobre varios departamentos, entre ellos, el más grande de la unión, el de Santa Cruz.

Este departamento recibe una gran influencia de la agroindustria de la soja y del sector de azúcar y alcohol de Brasil por su proximidad a los estados de Mato Grosso y Mato Grosso do Sul (incluso siendo acuñado de "Mato Grosso del Oeste"), ya que la extracción se convierte en una herramienta necesaria para los grandes empresarios y agricultores, incluidos los brasileños, que compran tierras fertiles para la producción en Bolivia, asegurando la expansión de la producción y la exportación a los principales importadores mundiales como, por ejemplo, a China.

En ese sentido, Zibechi sostiene que:

[...] el subimperialismo no es un fenómeno exclusivamente económico. No sólo exporta manufacturas sino también capital y, más importante aún desde este punto de vista, entra en la rapiña de recursos naturales, materias primas y fuentes de energía (ZIBECHI, 2012, p. 35).

\begin{tabular}{l|l}
\hline 78 & Ruy Mauro Marini y el Subimperialismo...
\end{tabular} 
Por lo tanto, otra etapa de los intereses brasileños en Bolivia se refiere a la operación y el control de las fuentes de energía como el petróleo y el gas con la importante participación de la estatal petrolera de Brasil, Petrobras (detiene el 35\% de la operación de los campos de Sábalo y San Alberto, los más grandes del país) (GANDARILLAS, 2014, p. 105); y minerales con la presencia de mineras como la Vale, Votorantim, Gerdau, entre otras.

En ese sentido, para que los recursos explotados se puedan exportar, se hace necesaria la construcción de obras de infraestructura en el país, que se pueden observar principalmente por la cantidad de nuevos caminos, en proyecto y ya construidos.

Con el fin de conectar las diferentes regiones, y sobre la base de que la deficiente infraestructura requiere mejores condiciones, se hacen concesiones a empresas extranjeras, teniendo Brasil un papel importante, así como la ejecución de carreteras, puentes y otras infraestructuras, con presencia de las empresas constructoras brasileñas como la OAS, Odebrecht, Camargo Correa, Andrade Gutierrez, entre otras, en virtud de los incentivos de financiación del Banco Nacional de Desarrollo Económico y Social (BNDES) y que tiene su institucionalización realizados por los hitos de la IIRSA.

En el polémico caso de la construcción de la interconexión vial de las regiones de Villa Tunari y San Ignacio de Moxos, atravesando el Territorio Indígena y Parque Nacional Isiboro-Secure (TIPNIS) en Bolivia, García Espinosa dice que:

La construcción está a cargo de la empresa brasileña OAS Ltda., con la cual se ha hecho un contrato llave en mano. Según este contrato "la empresa contratista se hace cargo del diseño y la construcción de la carretera a su cuenta y riesgo, por lo que no tiene supervisión". Se financia en un $80 \%$ con un crédito del Banco Nacional de Desenvolvimiento Económico y Social (BNDES) del Brasil y en un $20 \%$ con una contraparte provista 
por las gobernaciones de Cochabamba y Beni. De acuerdo a las condiciones con las que ha sido otorgado el crédito, todos los insumos para la construcción de la carretera son traídos del Brasil (ESPINOSA GARCÍA, 2011, p. 16).

De esa forma, la combinación de los factores mercado externo, Estadoy el consumo suntuoso resaltados por Marini para la composición del esquema de realización del subimperialismo, son articulados por los intereses de la alta burguesía brasileña que estimula la expansión de sus empresas a los mercados regionales y, articulada con el Estado, garantiza la expansión de los créditos del BNDES, mediante el argumento de la necesidad de vincular las regiones desconectadas de América del Sur.

La dinámica de control establecida por las empresas brasileñas se da bajo tres formas: fusiones, adquisiciones e inversiones directas (exportaciones de capital); importación de energía barata, estimulando la construcción de nuevos megaproyectos como la represa de Cachuela Esperanza en Bolivia y través de la reconfiguración del territorio suramericano, para que sirva a los intereses de la exportación de mercancías brasileñas a los grandes mercados del Pacífico (carretera Santos-Arica, en el extremo norte de Chile, pasando por territorio boliviano) (LUCE, 2010, p. 4-5).

Otro factor que permitió el proceso expansionista brasileño, aunque siga estando sometido al capital financiero internacional, fue la legitimación de este proyecto por los diplomáticos y los formuladores de las políticas del gobierno de Lula. De hecho, hubo un cuidado del Ministerio de Asuntos Exteriores para que la imagen de Brasil no esté vinculada a un subimperialismo y fortalecer la idea de la cooperación Sur -Sur, la importancia de Brasil para la integración regional en América del Sur, en la construcción de la dirección conjunta permitiendo el desarrollo de todos los países.

Sin embargo, Luce (2010) ilustra la advertencia del ministro Celso Amorim de que Brasil reduciría los préstamos del BNDES 
para la compra de tractores si algo le pasara a las propiedades de los terratenientes brasileños en el departamento de Santa Cruz, cuando se estaba debatiendo la reforma agraria en Bolivia.

De hecho, los breves ejemplos presentados nos permiten sintetizar que el tipo de integración regional que propone Brasil a Bolivia refleja una combinación de múltiples intereses de diferentes sectores de la burguesía brasileña, mediante un proyecto económico neodesarrollista, con respaldo de su política exterior para la construcción de la imágen de un Brasil Potencia, pero que en la práctica se demuestra mucho más cerca de una posición subimperialista que de un liderazgo solidário que vise superar la dependencia histórica en el continente.

\section{El caso paraguayo}

En la presente sección son presentados algunos elementos que permiten una primera aproximación a la comprensión de la proyección del subimperialismo brasileño en Paraguay. Aquí son presentados de forma general algunos datos, un análisis más detallado puede encontrarse en Carnebia López (2015).

En primer lugar, se percibe que durante el período 19982011 se produce una reconfiguración de la estructura productiva paraguaya, a lo que Osorio (2012) denominará patrón de exportación de especialización productiva, siendo que en este caso pareciera que el mismo se concentra en tres elementos claves: la soja, la energía eléctrica y la carne.

Este proceso es continuación y desdoblamiento de un proceso de mayor aliento que comienza en las décadas de 1960-70. En ese sentido, desde 1961 a la fecha se ha expandido significativamente la extensión de tierras destinadas al cultivo de soja y con ello, se produjo un también significativo crecimiento del volumen de producción de soja. Un elemento a tener en cuenta sobre esto es que al cruzar los datos de las extensiones cultivadas y el volumen producido, se constata 
que el rendimiento por hectárea - es decir la productividad - no ha sufrido grandes variaciones en el período analizado (CARNEBIA LÓPEZ, 2015, p. 25). Asimismo, si comparado con el rendimiento de países como Estados Unidos y Brasil, Paraguay presenta niveles de productividad bajos, lo que podría ser un indicativo de la existencia de transferencia de valor

Por su parte, el sector agrícola presenció una transformación, lo que Rojas (2009) coloca como un pasaje de la agri-cultura al agronegocio con la instalación del complejo agroindustrial. En este sentido, el proceso de migración de brasileños hacia Paraguay y el surgimiento del fenómeno brasiguayo, el cual como fuera señalado representa un complejo entramado de relaciones sociales y no puede ser considerado como un grupo social con características e intereses homogéneos. Sin embargo, según la bibliografía levantada, existe una camada de esos brasiguayos que consiguen una fuerte proyección tanto económica como social y política (ALBURQUERQUE, 2011; GERMANI, 2003; GLAUSER, 2012; PALAU, 2012).

Sobre esto, Glauser (2012) constata que el $50 \%$ de las 28 empresas "nacionales" vinculadas al agronegocio están en manos de brasileños y brasiguayos. Asimismo, según coloca el propio Glauser, estos productores se encuentran estrechamente vinculados a las grandes empresas transnacionales que son las responsables por la provisión de insumos (tales como semillas, agroquímicos/fertilizantes, entre otros), el acopio, industrialización y la distribución final.

Otro elemento que se constituye como un trazo importante a la hora de analizar la proyección del subimperialismo en Paraguay, es la energía y, particularmente, la usina hidroeléctrica de Itaipu, la cual es responsable por alrededor de $80 \%$ de la capacidad instalada de energía eléctrica en Paraguay. En ese sentido, vale señalar que la exportación de energía eléctrica supera ampliamente al consumo, representando la primera entre $60 \%$ y $70 \%$ de la capacidad instalada (CARNEBIA LÓPEZ, 2015, p. 68).

82 Ruy Mauro Marini y el Subimperialismo... 
Asimismo, pese a los intentos paraguayos por revertirlo, la energía producida por Itaipu sólo puede ser consumida por Brasil o Paraguay, a partir de lo cual se infiere que de la energía eléctrica que Paraguay exporta - considerando que durante gran parte del período 1998-2011 este constituye el principal producto de exportación (CARNEBIA LÓPEZ, 2015, p. 65) - la mayoría tiene como destino Brasil. Lo que, dadas las condiciones de funcionamiento de la entidad binacional, le garantiza a Brasil una significativa fuente de energía a bajo costo.

La energía eléctrica ha disminuido su peso en el total de las exportaciones, pero ello no se debe a una disminución de la producción de la misma, sino al gran crecimiento de las exportaciones de productos como la soja y la carne, lo cual permite reforzar la idea del avance del complejo agroindustrial sobre la economía paraguaya.

Al cruzar los datos referentes a las exportaciones con destino a Brasil y las importaciones provenientes de este, se encuentra un elemento que permite considerar como válida la concepción de la existencia de una división regional del trabajo: en cuanto Paraguay exporta a Brasil productos primarios (principalmente energía eléctrica $\mathrm{y}$ productos vegetales), importa de este maquinaria y insumos (CARNEBIA LÓPEZ, 2015, p. 75). Dado lo colocado hasta aquí, se podría inferir un proceso de transferencia de valor desde Paraguay hacia Brasil.

Por último se destaca que si bien el stock de IED en la economía paraguaya no es significativo en proporción del PIB, su evolución presenta un rasgo interesante y es que Brasil representa el segundo país más importante como fuente de origen de IED y, asimismo, se constata un crecimiento a partir de 2006, lo cual podría señalar un papel creciente del BNDES como financiador de los capitales -brasileños o no- pero que se proyectan desde este país hacia Paraguay.

Estos elementos colocados, al rescatarmos previamente el aporte de Ruy Mauro Marini para la comprensión de la realidad local en el 
siglo XXI, son apuntes preliminares que más que respuestas conclusivas y definitivas sobre la cuestión del subimperialismo brasileño en Bolivia y Paraguay, señalan algunos de los posibles trazos esenciales de dicho fenómeno, contribuyendo de alguna forma para ampliar los debates.

\section{Bibliografia}

ALBURQUERQUE, José Lindomar C. Fronteiras em movimento: Os brasiguaios na região da Tríplice Fronteira. In: MACAGNO, Lorenzo et al. A tríplice fronteira: espaços nacionais e dinâmicas locais. Curitiba: Editora UFPR, 2011.

ARISMENDI, Marco Octavio R. La amenaza del IIRSA en Bolivia. La Paz: LIDEMA, 2008.

CARNEBIA LÓPEZ, Fernando Matias. Para un análisis concreto de la realidad concreta: el subimperialismo brasileño en Paraguay en cuestión - apuntes preliminares. 2015. 97p. Trabajo de Conclusión de Curso (Graduación en Ciencias Económicas - Economía, Integración y Desarrollo) - Universidad Federal de Integración Latinoamericana, Foz do Iguaçu, 2015.

CÉSAR, Gustavo Rojas de Cerqueira. Intregración productiva Paraguay-Brasil: Nuevos Pasos en el Relacionamiento Bilateral. In: PRADO, Henrique Sartori de Almeida; NETO, Tomaz Espósito. Fronteiras e relações internacionais. Curitiba: Editora Íthala, 2015. Disponible en: <http://www.cadep.org.py/ uploads/2015/06/Para-WEB-CADEP.pdf $>$. Consultado el 03 dic. 2015.

ESPINOSA GARCÍA, Oscar. Carretera Villa Tunari-San Ignacio: Aspectos técnicos, económicos y legales, Revista Tecnología, Investigación y Docencia - Universidade Mayor de San Andrés, v.6, n.6, 2011. 
FERREIRA, Carla et al. Padrão de reprodução do capital: contribuições da teoria marxista da dependência. $1^{a}$ Edición. São Paulo: Boitempo Editorial, 2012.

GANDARILlAS G., Marco A. Bolivia: La década dorada del extractivismo. In: GANDARILLAS G., Marco A.(Coord.). Extrativismo: Nuevos contextos de dominación y Resistencias. Cochabamba: Centro de Documentación y Información Bolivia - CEDIB, 2014.

GERMANI, Guiomar Inez. Expropiados. Terra e água: o conflito de Itaipu. Salvador: EDUFBA - ULBRA, 2003.

GLAUSER, Marcos. Extranjerización del territorio paraguayo. Asunción: S/D, 2009.

LENIN, Vladimir Ilich. Imperialismo: estágio superior do capitalismo. $1^{\circ}$ Edição. São Paulo: Expressão Popular, 2012.

LUCE, Mathias Seibel. A Teoria do Subimperialismo em Ruy Mauro Marini: contradições do capitalismo dependente e a questão do padrão de reprodução do capital. A história de uma categoria. 2011. 225p. Tese (Doutorado) - Programa de Pós--Graduação em História, Instituto de Filosofia y Ciências Humanas da Universidade Federal de Rio Grande do Sul, 2011.

- O subimperialismo brasileiro revisitado: a política de integração regional do governo Lula (2003-2007). 2007. 136p. Dissertação (Mestrado) - Programa de Pós-graduação em Relações Internacionais, Instituto de Filosofia y Ciências Humanas da Universidade Federal de Rio Grande do Sul, 2007. 
MARINI, Ruy Mauro. América Latina: dependência e integração. 1a Edición. São Paulo: Editora Brasil Urgente, 1992.

. Dialéctica de la dependencia. Disponible en: $<\mathrm{http}: / / \mathrm{www}$.mariniescritos.unam.mx/004_dialectica_es.htm>. Consultado el: 19 jul. 2013.

_La acumulación capitalista mundial y el subimperialismo. Disponible en: <http://www.marini-escritos.unam.mx/006_ acumulacion_es.htm>. Consulta el: 16 dic. 2013.

. O ciclo do capital na economia dependente. In. FERREIRA, Carla et al. Padrão de reprodução do capital: contribuições da teoria marxista da dependência. 1ํㅡㄹ Edición. São Paulo: Boitempo Editorial, 2012.

.Subdesenvolvimento e revolução. $4^{\mathrm{a}}$ Edición. Florianópolis: Insular, 2013.

MARX, Karl. El Capital. t. 1, v. 1. 26 Edición. México DF: Siglo XXI Editores, s. a. de c. v., 2005.

PALAU VILADESAU, Tomás. Es lógico que una sociedad agredida se defienda. Recopilación de artículos 2008-2011. Asunción: Arandurã Editorial, 2012.

PRECIADO, Jaime. América Latina no Sistema-Mundo: questionamentos e alianças centro-periferia. Caderno CRH, v. 21, p. 253-268, 2008. 
OSORIO, Jaime. El nuevo patrón exportador de especialización productiva en América Latina, Revista da Sociedade Brasileira de Economia Política, n`31, São Paulo, 2012. Disponible en: <http:// www.sep.org.br/revista/download?id=220>. Consultado el: 10 dic. 2015.

QUIROGA, Marcelo. Santa Cruz, Oleocracia o Patria. México, D. F.: Siglo XXI, 1982.

RAMANZINI JR., H. e VIGEVANI, T. Pensamiento brasileño y integración regional. In: BRICEÑO RUIZ, José; CASAS GRAGEA, Ángel M.; RIVAROLA PUNTIGLIANO, Andrés (eds.). Integración latino-americana y caribenha: política y economía. México: Fondo de Cultura Económica de España, 2012.

ROJAS VILLAGRA, Luis. Actores del agronegocio en Paraguay. Asunción: Arandurã Editorial, 2009.

TRASPADINI, Roberta; STEDILE, João. Ruy Mauro Marini: Vida e obra. 2ª Edición. São Paulo: Expressão Popular, 2011.

VUYK, Cecilia. Subimperialismo brasileño y dependencia del Paraguay. Los intereses económicos detrás del Golpe de Estado de 2012. Paraguay: Arandurã Editorial, 2014.

ZIBECHI, Raúl. Brasil Potencia. Entre la integración regional y un nuevo imperialismo. Málaga: Baladre/Zambra, 2012. 
